\title{
On the quasi-stratified algebras of Liu and Paquette
}

\author{
W.D. Burgess ${ }^{1}$ \\ Department of Mathematics and Statistics, \\ University of Ottawa, Ottawa, ON K1N 6N5 \\ A. Mojiri \\ Department of Mathematics,Texas A\&M University-Texarkana, \\ Texarkana, TX 75505-5518
}

Key words: Artin algebra, quasi-stratified, quasihereditary, left serial.

Mathematics Subject Classification: 16G10, 16G20, 16F10.

\begin{abstract}
S.-P. Liu and C. Paquette defined a class of artin algebras, more general than the standardly stratified ones, called quasi-stratified algebras. Not only is the Cartan Determinant Conjecture (CDC) true for these algebras, so is its converse. This article shows that this class of algebras is preserved under "pruning" sources and sinks from the left quiver. It compares the classes of quasi-stratified and left serial algebras, as well as quasi-stratified and gentle algebras. Holm has shown that the CDC holds for gentle algebras; the converse is also established. It is shown when a Yamagata family of algebras of large finite global dimension yield quasi-stratified ones and constructs quasi-stratified elementary algebras from smaller ones.
\end{abstract}

\section{Introduction.}

All rings considered will be artin algebras; in some cases we will specialize to finite dimensional algebras over a field. For such an algebra $A$, we fix a complete orthogonal set of primitive idempotents, $\left\{e_{1}, \ldots, e_{n}\right\}$. The radical is denoted $\mathbf{J}(A)$, or just $\mathbf{J}$. Some of the results involving left quasi-stratified rings could be proved for general left artinian rings but for reasons of economy the stronger hypothesis will be imposed. It will be seen in an appendix that the class of quasi-stratified algebras is closed under Morita equivalence and, hence, we will usually assume in proofs that our algebras are basic. All the proofs below could be adapted to non-basic algebras.

The (left) Cartan matrix of a basic algebra $A, C(A) \in \mathbf{M}_{n}(\mathbf{Z})$, has $i, j$ entry the number of copies of $A e_{i} / J e_{i}$ in a composition series of $A e_{j}$. The Cartan determinant, $\operatorname{cd} A=\operatorname{det} C(A)$. There is a right version but, for artin algebras, the two Cartan determinants are equal (e.g., Fuller (1992, Proposition 1.2)). A result of Eilenberg (see Fuller (1992, Theorem 1.1)) says that if gl. dim. $A<\infty$ then $\mathrm{cd} A= \pm 1$. The Cartan determinant conjecture asserts that if gl. dim. $A<$ $\infty$ then $\operatorname{cd} A=1$. The survey Fuller (1992) shows the state of the conjecture up to 1992.

The algebras, quasi-stratified algebras, (definitions are in Section 1) studied by S. Liu and C. Paquette (Liu \& Paquette (2006)) have the property that not

\footnotetext{
${ }^{1}$ The first author acknowledges the support of a grant from the NSERC. Both authors thank Kent Fuller for some helpful suggestions, especially about the Morita invariance. For correspondence: wburgess@uottawa.ca
} 
only does the Cartan determinant conjecture hold for them (since their Cartan determinants are always strictly positive) but so does its converse; i.e., the global dimension is finite if and only if the Cartan determinant is 1 . These algebras are filtered by chains of projective ideals with the innovation that an ideal in the chain may be projective on the left or on the right, and these ideals need not be idempotent. The aims of this article are to find families of examples of quasi-stratified algebras, to relate them to other interesting classes such as left serial and gentle algebras, and to present constructions.

The article is divided into sections. The first shows that questions about quasi-stratified algebras may be reduced to those about algebras whose (left) quiver has neither sources nor sinks. This is then used in Section 2 to show that in a left serial algebra if the vertices in the quiver which are sources are removed (as in Section 1) we then can look at an algebra which is a division algebra or whose quiver is an oriented cycle. These latter algebras are here called left serial of serial type. A left serial algebra $A$ of serial type is left quasi-stratified if and only if it contains a primitive idempotent $e$ where ${ }_{A} A e A$ is projective. From this, a left serial algebra is quasi-stratified if and only if it is left standardly stratified. Section 3 looks at the families of algebras constructed in Yamagata (1994), all $A_{n}$ in a family are shown to be quasi-stratified, in fact, ultimate-hereditary, the quasi-stratified algebras of finite global dimension (Definition 1.2), if the seed algebra $A_{0}$ is hereditary. The main purpose of Section 4 is to show that new, more complex, quasi-stratified elementary algbras over a field can be built from an existing one. There are two constructions. Section 5 looks at monomial algebras. A characterization is given of when the ideal generated by a path is left or right projective. This result is specialized to the case of quadratic monomial algebras, monomial algebras whose relations are generated by paths of length 2. If $A$ is quadratic monomial and gl. $\operatorname{dim} . A<\infty$ then $A$ is left and right ultimate-hereditary. Then a theorem of Holm is used to show that a gentle algebra satisfies the converse of the Cartan determinant conjecture. A brief sixth section uses a result of Colby to look at endomorphism rings of tilting and cotilting modules over a quasi-stratified algebra. The paper ends with an appendix where a sketch is given of a proof showing that the class of quasi-stratified algebras is closed under Morita equivalence.

\section{Definitions and quiver reductions.}

Idempotent ideals which are projective on one side have been extensively studied (see Dlab \& Ringel (1989)); the article Liu \& Paquette (2006) extends this study to ideals which are not necessarily idempotent.

Definition 1.1 (Liu \& Paquette (2006)). (i) An ideal I of an algebra $A$ is called projective if ${ }_{A} I$ or $I_{A}$ is projective. Given an ideal $I$ of $A$, for some $k \geq 1, I^{k}=I^{k+1}$. Then, $I^{k}$ is called the idempotent part of $I$. (ii) A projective ideal $I$ is called quasi-stratifying if its idempotent part has the form AeA where $e=0$ or $e$ is a primitive idempotent. If, in addition, $e \boldsymbol{J} e=\mathbf{0}, I$ is called quasi-heredity.

Definition 1.2 (Liu \& Paquette (2006)). (i) An algebra $A$ is called quasi- 
stratified if it has a quasi-stratifying chain, i.e., a chain of ideals

$$
\mathbf{0}=I_{0} \subset I_{1} \subset \cdots \subset I_{t-1} \subset I_{t}=A
$$

so that, for $j=1, \ldots t, I_{j} / I_{j-1}$ is a quasi-stratifying ideal in $A / I_{j-1}$.

(ii) If $A$ is quasi-stratifying with a quasi-stratifying chain as above where each $I_{j} / I_{j-1}$ is quasi-heredity, then $A$ is called ultimate-hereditary.

(iii) If all the ideals $I_{j} / I_{j-1}$ in (i) or (ii) are projective on the left (right) then the algebra is called left (right) quasi-stratified or left (right) ultimatehereditary.

The paper Liu \& Paquette (2006) has much more information about the algebras defined above than can be quoted here, the key results for this note, Liu \& Paquette (2006, Proposition 1.3, Corollary 1.4, Proposition 2.1, Corollary 2.3 and Theorem 2.5), are assembled in the following.

Theorem 1.3 (Liu \& Paquette (2006)). Let $A$ be an algebra and $I$ a projective ideal with idempotent part $A e A, e^{2}=e$ and $t$ minimal with $I^{t}=$ AeA.

(I) gl. $\operatorname{dim} . A / I \leq$ gl. $\operatorname{dim} . A+2(t-1)$ and gl. $\operatorname{dim} . e A e \leq$ gl. $\operatorname{dim} . A \leq$ gl. dim. $e A e+$ gl. $\operatorname{dim} . A / I+2$; hence, gl. $\operatorname{dim} . A<\infty$ if and only if gl. dim. $e A e<$ $\infty$ and gl. $\operatorname{dim} . A / I<\infty$ (by convention gl. $\operatorname{dim} . \mathbf{0}=-1)$.

(II) $\operatorname{cd} A=(\operatorname{cd} e A e)(\operatorname{cd} A / I)$ (by convention $\operatorname{cd} \mathbf{0}=1)$.

(III) (i) If $A$ is quasi-stratified then $\operatorname{cd} A>0$; and,

(ii) the following are equivalent for a quasi-stratified algebra $A$ :

(a) $\operatorname{cd} A=1$, (b) gl. $\operatorname{dim} . A<\infty$ and (c) $A$ is ultimate-hereditary.

The left quiver $Q_{l}(A)$ of a basic left artinian ring $A$ has $r$ arrows from $i$ to $j$ if $\mathbf{J} e_{i} / \mathbf{J}^{2} e_{i}$ has $r$ copies of $A e_{j} / \mathbf{J} e_{j}, 1 \leq i, j \leq n$. The right quiver $Q_{r}(A)$ is defined similarly. If $i$ is a vertex in $Q_{l}(A)$ so that there are no arrows entering $i$ except possibly loops at $i$, then $i$ is called an $l$-source. An l-sink is defined similarly. In general, if $i$ is an l-source in $Q_{l}(A)$ then $A e_{i} A=A e_{i}$ is projective (recall that our algebras are assumed to be basic) and if $i$ is an l-sink in $Q_{l}(A)$, ${ }_{A} A e_{i} A$ is semisimple and $A e_{i} A=e_{i} A$ is projective as a right module. The initial and terminal vertices of a path $p$ in $Q_{l}(A)$ are denoted, respectively, $s(p)$ and $t(p)$.

For future reference we record the following two simple lemmas and a corollary. The corollary is a special case of Fuller (1992, Proposition 2.3).

Lemma 1.4. Let $I$ be an ideal in a basic algebra $A$. (i) If I is nilpotent, $Q_{l}(A)$ and $Q_{l}(A / I)$ have the same vertices. (ii) If $I$ is generated by a primitive idempotent, say $I=A e_{1} A$, then the vertices of $Q_{l}(A / I)$ are $2, \ldots, n$. (iii) If $A e_{1} A$ is the idempotent part of $I$ then the vertices of $Q_{l}(A / I)$ are $2, \ldots, n$.

Lemma 1.5. Let $e$ be an idempotent of an algebra $A$ so that $A e A=A e$ or $A e A=e A$ and $K$ an ideal. Then $A /(K+A e A) \cong(1-e+K)(A / K)(1-e+K)$.

Proof. When $A e A=A e$, the isomorphism is given by $\theta: a+(K+A e A) \mapsto$ $(1-e) a(1-e)+K$. 
Corollary 1.6. Let e be a primitive idempotent in a basic algebra $A$ such that $e$ corresponds to an l-source or an l-sink in $Q_{l}(A)$. Then, $Q_{l}((1-e) A(1-e))$ is obtained from $Q_{l}(A)$ by removing the corresponding vertex and the arrows to or from it. When the vertex is an l-source, $A e A=A e$ is projective. When the vertex is an l-sink in $Q_{l}(A), A e A=e A$ is projective.

Theorem 1.7. Let $A$ be a quasi-stratified algebra whose quiver has more than one vertex and with quasi-stratification

$$
\mathbf{0}=I_{0} \subset I_{1} \subset \cdots \subset I_{t-1} \subset I_{t}=A .
$$

Suppose that 1 is an l-source or an l-sink in $Q_{l}(A)$. Then, the proper inclusions of

$$
\mathbf{0}=I_{0} \subset A e_{1} A \subseteq I_{1}+A e_{1} A \subseteq \cdots \subseteq I_{t-1}+A e_{1} A \subseteq I_{t}=A
$$

form a quasi-stratification of $A$.

Proof. We may assume, using Theorem 7.3, below, that $A$ is basic. We will prove that case where 1 is an l-source and, since $A$ is basic, we have $A e_{1} A=A e_{1}$. It needs to be shown that, for $j=1, \ldots, t, \frac{I_{j}+A e_{1} A}{I_{j-1}+A e_{1} A}$ is projective on one side as an $\frac{A}{I_{j-1}+A e_{1} A}=\tilde{A}$-module, if $I_{j}+A e_{1} A \neq I_{j-1}+A e_{1} A$. There are two cases where we write $A / I_{j-1}=\bar{A}$. We will identify $\tilde{A}$ with $\left(1-\bar{e}_{1}\right) \bar{A}\left(1-\bar{e}_{1}\right)$ using Lemma 1.5.

Case 1: $\bar{A} \bar{I}_{j}$ is projective. For each $k \neq 1, \bar{I}_{j} \bar{e}_{k}$ is a direct sum of indecomposable left $\bar{A}$-projective modules each isomorphic to $\bar{A} \bar{e}_{s}$, for some $s \neq 1$ (since $\bar{A} \bar{e}_{1} \bar{A} \bar{e}_{k}=\mathbf{0}$ when $\left.k \neq 1\right)$. From $\bar{I}_{j} \bar{e}_{k}\left(1-\bar{e}_{1}\right)=\bar{I}_{j} \bar{e}_{k}$ we have $\left(1-\bar{e}_{1}\right) \bar{I}_{j} \bar{e}_{k}\left(1-\bar{e}_{1}\right)$ is isomorphic to a direct sum of indecomposable projective left modules of the form $\left(1-\bar{e}_{1}\right) \bar{A} \bar{e}_{s}\left(1-\bar{e}_{1}\right)$, some $s \neq 1$. Hence, $\left(1-\bar{e}_{1}\right)\left(\bar{I}_{j}+\bar{A} \bar{e}_{1} \bar{A}\right)\left(1-\bar{e}_{1}\right)$ is left projective.

Case 2: $\left(\bar{I}_{j}\right)_{\bar{A}}$ is projective. For $k \neq 1, \bar{e}_{k} \bar{I}_{j}$ is a direct sum of indecomposable projective right $\bar{A}$-modules of the form $L=\bar{e}_{k} \bar{u} \bar{e}_{s} \bar{A}, \bar{u} \in \bar{A}$ and $1 \leq s \leq n$. Then, $\left(1-\bar{e}_{1}\right) L\left(1-\bar{e}_{1}\right)=\bar{e}_{k} \bar{u} \bar{e}_{s} \bar{A}\left(1-\bar{e}_{1}\right)$, which is a projective right $\left(1-\bar{e}_{1}\right) \bar{A}\left(1-\bar{e}_{1}\right)-$ module (it is $\mathbf{0}$ if $s=1$ ).

We next need to verify that each proper inclusion gives a quasi-stratifying ideal. If $I_{j}+A e_{1} A$ is nilpotent modulo $I_{j-1}+A e_{1} A$, there is nothing to be done. Otherwise, it needs to be shown that the idempotent part of $I_{j}+A e_{1} A$ is generated by a primitive idempotent modulo $I_{j-1}+A e_{1} A$. Since $\left(I_{j}+A e_{1} A\right)^{k}=$ $\left(I_{j}\right)^{k}+A e_{1} A$, we may assume that, for some $r$ and some primitive idempotent $\bar{e} \in \bar{A}$, that $\left(\bar{I}_{j}\right)^{r}=\bar{A} \bar{e} \bar{A}$ is the idempotent part of $\bar{I}_{j}$ and that $\left(I_{j}+A e_{1} A\right)^{r}$ is the idempotent part of $I_{j}+A e_{1} A$, modulo $I_{j-1}+A e_{1} A$.

For some $i>1, \bar{A} \bar{e} \bar{A}=\bar{A} \bar{e}_{i} \bar{A}$ (by Lemma 1.4 (iii)). By Lemma 1.4 (ii) $\bar{e}_{i}$ is a primitive idempotent in $\bar{A} / \bar{A} \bar{e}_{1} \bar{A}=A /\left(I_{j-1}+A e_{1} A\right)$.

If one wished a proof of Theorem 1.7 without assuming $A$ basic, the idempotent $e_{1}$ would be replaced by the sum of the primitive idempotents equivalent to it. The above proof works for any idempotent $E$ with $A E A=A E$ (or $A E A=E A$ ) where there is a quasi-stratifying chain of ideals of $A$ leading up to $A E A$; however, there is no obvious application of the more general form. 
Definition 1.8. If $A$ is a basic algebra whose quiver has more than one vertex and is such that $Q_{l}(A)$ has an l-source, say 1 , then passing to $\left(1-e_{1}\right) A\left(1-e_{1}\right)$ is called pruning an l-source. Similarly for an l-sink.

Corollary 1.9. Let $A$ be a basic algebra. There is an algebra $B$ so that $Q_{l}(B)$ has only one vertex or $Q_{l}(B)$ has neither l-sources nor l-sinks and $A$ is quasistratified if and only if $B$ is quasi-stratified.

Proof. An algebra $B_{1}$ is obtained from $A$ by a sequence of steps of pruning an l-source from the previous algebra in the sequence so that $Q_{l}\left(B_{1}\right)$ has only one vertex or $Q_{l}\left(B_{1}\right)$ has no l-sources. An algebra $B$ is obtained from $B_{1}$ by a sequence of steps of pruning an l-sink from the previous algebra in the sequence so that $Q_{l}(B)$ has only one vertex or $Q_{l}(B)$ has neither l-sources nor l-sinks.

We may renumber the vertices of $Q_{l}(A)$ so that the vertices (if any) removed in passing to $B_{1}$ are, in order, $1, \ldots, m_{1}$, and those (if any) removed in passing from $B_{1}$ to $B$ are $m_{1}+1, \ldots, r$. There is nothing to prove if no vertices are removed and it is assumed that $r>0$. We then put $\varepsilon_{i}=e_{1}+\cdots+e_{i}$, for $i=1, \ldots, r$ and $\varepsilon_{0}=0$. By a repeated application of Lemma 1.5 we have that $\tau: A \rightarrow\left(1-\varepsilon_{r}\right) A\left(1-\varepsilon_{r}\right)$, given by $\tau(a)=\left(1-\varepsilon_{r}\right) a\left(1-\varepsilon_{r}\right)$, is a ring surjection.

Suppose that $\mathbf{0}=I_{0} \subset I_{1} \subset \cdots \subset I_{k}=B$ is a quasi-stratification of $B$. Then,

$$
\mathbf{0}=A \varepsilon_{0} A \subset A \varepsilon_{1} A \subset \cdots \subset A \varepsilon_{r} A \subset \tau^{-1} I_{1} \subset \cdots \subset \tau^{-1} I_{k}=A
$$

is a quasi-stratification of $A$. Conversely, if $\mathbf{0}=I_{0} \subset I_{1} \subset \cdots \subset I_{k}=A$ is a quasi-stratification of $A$ then, using repeated applications of Theorem 1.7, we see that the proper inclusions of

$$
\mathbf{0}=A \varepsilon_{0} A \subset A \varepsilon_{1} A \subset \cdots \subset A \varepsilon_{r} A \subseteq I_{1}+A \varepsilon_{r} A \subseteq \cdots \subseteq I_{k}+A \varepsilon_{r} A=A
$$

form a quasi-stratification of $A$. Dividing by $A \varepsilon_{r} A$ gives a quasi-stratification of $A / A \varepsilon_{r} A=B$.

The proof of Corollary 1.9 also shows that "quasi-stratified" may be replaced by "left (or right) quasi-stratified"; if sources and sinks are used instead of lsources and l-sinks, the proof also works for "(left or right) ultimate-hereditary".

Along the same lines as Fuller (1992, Corollary 2.13), we have the first part of the following. The irreducible components are algebras of the form $E_{j} A E_{j}$, where $E_{j}$ is the sum of the primitive idempotents corresponding to an irreducible component (also called a strongly connected component) of $Q_{l}(A)$ (ibid, p. 55.)

Proposition 1.10. (i) Let $A$ be an algebra such that its irreducible components are quasi-stratified. Then, gl. $\operatorname{dim} .(A)<\infty$ if and only if $\operatorname{cd}(A)=1$.

(ii) Let $A$ be a quasi-stratified (an ultimate-hereditary) algebra, then the irreducible components of $A$ are also quasi-stratified (ultimate-hereditary).

Proof. (i) Suppose there are $s$ irreducible components, $E_{j} A E_{j}, j=1, \ldots, s$. According to Fuller (1992, Corollary 2.6), the global dimension of $A$ is bounded above by the sums of the global dimensions of the irreducible components plus 
$s-1$. Moreover, for each irreducible component $E_{j} A E_{j}, \operatorname{cd}\left(E_{j} A E_{j}\right)>0$ (Theorem 1.3 (III)) and gl. $\operatorname{dim} .\left(E_{j} A E_{j}\right)<\infty$ if and only if $\operatorname{cd}\left(E_{j} A E_{j}\right)=1$. Finally (Fuller (1992, Proposition 2.4)), $\operatorname{cd}(A)=\prod_{j=1}^{s} \operatorname{cd}\left(E_{j} A E_{j}\right)$.

(ii) Let $\mathbf{0} \subset I_{1} \subset \cdots \subset I_{t-1} \subset I_{t}=A$ be a quasi-stratifying chain for $A$. One of the idempotents, say $E_{j}$, is fixed as in part (i). It will suffice to show that the proper inclusions of $\mathbf{0} \subseteq E_{j} I_{1} E_{j} \subseteq \cdots \subseteq E_{j} I_{t-1} E_{j} \subseteq E_{j} A E_{j}$ form a quasi-stratifying chain. We reason as in Theorem 1.7 by noting that for $k \neq l$, $E_{k} A E_{l}=\mathbf{0}$ or $E_{l} A E_{k}=\mathbf{0}$. We put $\bar{A}=A / I_{i-1}$. It easy to see that if $\bar{I}_{i}$ has an indecomposable left projective component $\bar{L} \neq \mathbf{0}$, say $\bar{L}=\bar{A} \bar{e}_{l} \bar{u} \bar{e}_{k} \cong \bar{A} \bar{e}_{l}$ where right multiplication by $\bar{u}$ is an isomorphism, then $\bar{E}_{j} \bar{A} \bar{e}_{l} \bar{u} \bar{e}_{k} \bar{E}_{j}=\mathbf{0}$ if $\bar{e}_{l} \bar{E}_{j}=0$ or $\bar{e}_{k} \bar{E}_{j}=0$; otherwise,

$$
\bar{E}_{j} \bar{L} \bar{E}_{j}=\bar{E}_{j} \bar{A} \bar{E}_{j} \bar{e}_{l} \bar{u} \bar{e}_{k} \cong \bar{E}_{j} \bar{A} \bar{E}_{j} \bar{e}_{l}=\bar{E}_{j} \bar{A} \bar{E}_{j} \bar{e}_{l} \bar{E}_{j}
$$

an indecomposable projective $\bar{E}_{j} \bar{A} \bar{E}_{j}$-module. The method is similar for the case where $\bar{I}_{i}$ is right projective. If $\bar{E}_{j} \bar{I}_{i} \bar{E}_{j}$ is nilpotent we are done, otherwise let the idempotent part of $\bar{I}_{i}$ be $\bar{A} \bar{e}_{p} \bar{A}$. Then $\bar{E}_{j} \bar{e}_{p}=\bar{e}_{p}$ and the primitive idempotent $\bar{e}_{p}$ is in $\bar{E}_{j} \bar{I}_{i} \bar{E}_{j}$, and is the idempotent part of $\bar{E}_{j} \bar{I}_{i} \bar{E}_{j}$.

The final part about $A$ ultimate-hereditary follows from part (i) and Liu \& Paquette (2006, Theorem 2.5) (quoted in Theorem 1.3 (III) (ii)).

However, having the irreducible components quasi-stratified does not mean that the algebra is quasi-stratified. Example 2.5, below, will illustrate this.

\section{On left serial algebras.}

In this section, quasi-stratified serial and left serial algebras will be characterized. Recall that a connected algebra $A$ is one with no non-trivial central idempotents, i.e., $Q_{l}(A)$ is a connected graph. We will use some results originally stated for connected serial rings but which work equally well for left (or right) serial rings for which the left (right) quiver is either a path or an oriented cycle. The results in question are those stated entirely in terms of one-sided ideals. The restriction on the shape of the quiver means that these rings have Kupisch series (Anderson \& Fuller (1992, page 348)) on the appropriate side. In particular the left hand version of $i b i d$, Lemma 32.5 works here. In addition, all the results of Burgess \& Fuller (1989, Section 2) hold in this setting (as was pointed out at the end of that section). We give a name to the one-sided serial rings just described.

Definition 2.1. A connected left serial algebra $A$ is said to be of serial type if its left quiver is a path or an oriented cycle. The right hand version is defined similarly.

Proposition 2.2. Let $A$ be a connected left serial algebra of serial type.

(i) If $A$ has a quasi-stratifying ideal $I$, with ${ }_{A} I$ projective, then there is a primitive idempotent $e_{i}$ with ${ }_{A} A e_{i} A$ projective.

(ii) If $A$ is not quasihereditary and has a primitive idempotent e with ${ }_{A} A e A$ projective, then $A$ is of infinite global dimension. 
(iii) If $A$ is a serial algebra then (i) also applies with $I_{A}$ projective giving a primitive idempotent $e_{i}$ with $A e_{i} A_{A}$ projective. Moreover, if $e$ is a primitive idempotent of $A$ so that ${ }_{A} A e A$ is projective, then $A e A_{A}$ is projective, and conversely.

Proof. Once again it will be assumed that $A$ is basic. Throughout, if $x \in A$, right multiplication by $x$ will be denoted by $\rho_{x}$. For an integer $i,[i]$ means the smallest positive residue of $i$ modulo $n$.

(i) If $Q_{l}(A)$ has a source, say 1 , then $A e_{1}=A e_{1} A$ is projective. In the contrary case, $Q_{l}(A)$ is an oriented cycle whose vertices are numbered with arrows $i \rightarrow[i+1], i=1, \ldots, n$. If ${ }_{A} I$ is not nilpotent, its idempotent part gives what is required. Thus, we can assume that $\mathbf{0} \neq{ }_{A} I$ is nilpotent. We may renumber the idempotents cyclically so that $I e_{i} \cong A e_{n}$ for some $i, 1 \leq i<n$. It will be shown that ${ }_{A} A e_{n} A$ is projective.

As in Anderson \& Fuller (1992, Lemma 32.5), the arrows $j \rightarrow[j+1]$ are labeled $a_{j}=e_{[j+1]} a_{j} e_{j}$. Then, each $\mathbf{J}^{r} e_{j}=A a_{[j+r-1]} \cdots a_{j}$. With this notation, $I e_{i}=A e_{n} A e_{i}=A a_{n-1} \cdots a_{i}$. From this, $\rho_{a_{n-1} \cdots a_{i}}$ is one-to-one on $A e_{n}$. Hence, for $i \leq j<n, \rho_{a_{n-1} \cdots a_{j}}$ is one-to-one on $A e_{n}$. It follows that, for $i \leq j<n$, $A e_{n} A e_{j}$ is projective. If ${ }_{A} A e_{n} A$ is not projective, there is a maximal $j, 1 \leq$ $j<i$, with $A e_{n} A e_{j}$ not projective. However, $A e_{n} A e_{j} \subset I e_{j}$ since $A e_{n} A e_{j}=$ $I e_{i} a_{i+1} \cdots a_{j}$. Since $I e_{j}$ is projective, $I e_{j} \cong A e_{s}$, for some $s, s \neq j, n$, and $A e_{n} A e_{j} \subsetneq I e_{j}$. There are two cases to be considered.

(a) $j<s<n$ : Then $\rho_{a_{s+1} \cdots a_{j}}$ is one-to-one on $A e_{s}$ and, hence, also on $A e_{n} A e_{s} \cong A e_{n}$. This contradicts the choice of $j$.

(b) $1 \leq s<j$ : In this case,

$$
I e_{j}=A e_{s} A e_{j}=A a_{[s-1]} \cdots a_{i} \cdots a_{j}=A e_{s} A e_{n} a_{n-1} \cdots a_{i} \cdots a_{j} \subseteq A e_{n} A e_{j},
$$

which is not possible since we have $A e_{n} A e_{j} \subsetneq I e_{j}$.

This contradiction shows that ${ }_{A} A e_{n} A$ is projective, as required.

(ii) We know from Theorem 1.3 (II) that $\operatorname{cd}(A)=\operatorname{cd}(e A e) \operatorname{cd}(A / A e A)$. By Burgess \& Fuller (1989, Lemma 2.1 and Lemma 2.2), $A / A e A$ is quasihereditary, showing that $\operatorname{cd}(A / A e A)=1$ (Burgess \& Fuller (1989, Proposition 1.3)). However, since $A e A$ is not a heredity ideal, i.e., $e \mathbf{J} e \neq \mathbf{0}, \operatorname{cd}(e A e)>1$. Hence, $\operatorname{cd}(A)>1$, showing infinite global dimension.

(iii) We use Anderson \& Fuller (1992, Lemma 32.5) once more; it shows that the same elements $a_{i}$ (as in (i)) give the right structure of $A$. We may again assume that $Q_{l}(A)$ is an oriented cycle. Suppose that ${ }_{A} A e_{n} A$ is projective. This means

$(*)$ if $i \neq n$ and $a_{n-1} \cdots a_{i} \neq 0$ and $0 \neq x=x e_{n}$ then $x a_{n-1} \cdots a_{i} \neq 0$;

and we may take $x$ to be an appropriate product of the $a_{j}$. We need the right hand version of this to be true. We want each $e_{i} A e_{n} A=a_{[n-i]} \cdots a_{n} A$ to be projective. I.e., if $i \neq n$ and $a_{[i-1]} \cdots a_{n} \neq 0$ and $x=e_{n} x \neq 0$ then $a_{[i-1]} \cdots a_{n} x \neq 0$. We may take $x=a_{n-1} \cdots a_{j}$, where the indices of the factors may go through complete cycles, and we need to do an induction on the number of times $a_{n}$, call this $r$, occurs in the product for $x$. 
Case 1: $r=0$. Then, $a_{[i-1]} \cdots a_{n} x=a_{[i-1]} \cdots a_{n} \cdot a_{n-1} \cdots a_{j} \neq 0$ by $(*)$.

Case 2: We assume the statement for $r=t \geq 0$ and that the expression for $x$ has $t+1$ occurrences of $a_{n}$. Let $y$ be the product of all the factors of $x$, from the left, up to but not including the $t+1$ copy of $a_{n}$ with $x=$ $y a_{n} \cdots a_{j}$. The induction hypothesis gives $a_{[i-1]} \cdots a_{n} \cdot y \neq 0$. Now $(*)$ shows that $a_{[i-1]} \cdots a_{n} \cdot y \cdot a_{n} \cdots a_{j}=a_{[i-1]} \cdots a_{n} \cdot x \neq 0$.

We now have that $A e_{n} A_{A}$ is projective.

Burgess \& Fuller (1989, Proposition 2.3) says that a serial algebra $A$ is quasihereditary if and only if it has a heredity ideal; i.e., if and only if there is a primitive idempotent $e$ so that $A e A$ is a heredity ideal. This is generalized.

Corollary 2.3. A left serial algebra A of serial type is left quasi-stratified if and only it has a primitive idempotent e such that ${ }_{A} A e A$ is projective. Moreover, if $A$ is a serial algebra and is quasi-stratified it is both left and right quasi-stratified.

Proof. If $A$ is left quasi-stratified it has a quasi-stratifying ideal $I$ projective on the left. The proof of Proposition 2.2 (i) proceeds by showing that ${ }_{A} A e_{n} A$ is projective or that there is an idempotent $e_{j}$ with $e_{j} \in I$. Then $I$ is not nilpotent and its "idempotent part" (Liu \& Paquette (2006, Lemma 1.1)), $\mathbf{0} \neq A e A \subseteq I$ and $A e A$ is projective, as required.

In the other direction, $A / A e A$ is quasihereditary by Burgess \& Fuller (1989, Lemma 2.1 and Lemma 2.2). Hence, $A$ is left quasi-stratified.

The second statement follows from Proposition 2.2 (iii) because a quasihereditary algebra is both left and right quasi-stratified.

Proposition 2.2 (ii) and Corollary 2.3 also give: if $A$ is left serial of serial type and is left quasi-stratified then it is either quasihereditary or gl. dim. $A=\infty$.

Corollary 1.9 allows us to characterize left or right serial algebras which are left or right quasi-stratified, respectively.

Theorem 2.4. Let $A$ be a connected left serial algebra. There is a factor algebra $B$ of $A$ which is of serial type and is either a simple algebra or whose quiver is an oriented cycle such that $A$ is left quasi-stratified (quasihereditary) if and only if $B$ is left quasi-stratified (quasihereditary). The statements for right serial algebras are analogous.

Proof. We once again assume that $A$ is basic. The form of the left quiver of a connected left serial algebra is described, for example, in Burgess et al. (1985, page 160) or Fuller (1992, page 58); in particular, if the sources are pruned one by one, as in Corollary 1.9, the result is either a single vertex without loops or an oriented cycle. This shows that the process described in the statement can be carried out to arrive at an algebra $B$ as described in the statement. Then Corollary 1.9 says that $A$ is left quasi-stratified if and only if $B$ is. Moreover, the remark after Corollary 2.3 also shows that $A$ is quasihereditary if and only if $B$ is quasihereditary; this is because the ideals used in the pruning process are heredity ideals. 
The global assumption that our rings are artin algebras is essential in Theorem 2.4 since the example after Fuller (1992, Corollary 3.7) is of a right serial ring $R$ with $\operatorname{cd} R=1$ and gl. $\operatorname{dim} . R=\infty$. The right Cartan determinant is, however, 0 .

Recall from, e.g., Ágoston et al. (1998, Definition 1.3) the definition of a (left) standardly stratified algebra in terms of ideals generated by primitive idempotents. Then Theorem 2.4 also yields: A left serial algebra is left quasi-stratified if and only if it is standardly stratified. In one direction the ordering of the primitive idempotents follows the pruning of the sources and then Corollary 2.3 is used; the converse is true for all algebras.

It is easy to find an example of a serial algebra which is not quasihereditary but has a left projective ideal of the form $A e A, e$ primitive; it is, thus, quasistratified.

Example 2.5. The following diagrams define, over some field $K$, a serial algebra $A$ which is not quasihereditary (it is of infinite global dimension) but has a left projective ideal $A e_{1} A$ and thus is quasi-stratified. Moreover, the same algebra can be augmented to give an algebra $B$ whose irreducible components are quasi-stratified but B is not (cf. Proposition 1.10).

The algebra $A$ is the one with idempotents $e_{1}, e_{2}$ and $e_{3}$. The algebra $B$ has, in addition, $e_{4}$ and $e_{5}$. I.e., $A=\left(e_{1}+e_{2}+e_{3}\right) B\left(e_{1}+e_{2}+e_{3}\right)$. We have $\operatorname{cd}(A)=\operatorname{cd}(B)=2$. The irreducible components of $B$ are two quasi-stratified serial algebras. The diagrams, showing the left and right structures, make clear why $B$ has no quasi-stratifying ideals projective on the left or right. (There are simpler examples, of finite global dimension, which are not quasi-stratified but whose irreducible components are quasi-stratified.)

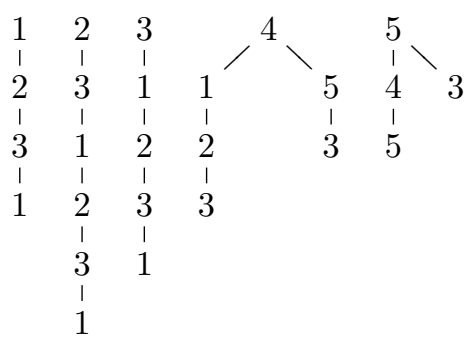

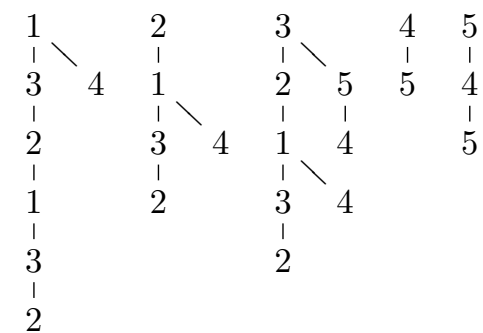

We next quote an example of a serial algebra of global dimension 4 which has no projective ideals of the form $A e A, e=e^{2}$ primitive. Hence, it is not quasi-stratified. Recall (Burgess \& Fuller (1989, Proposition 2.7)) that every serial algebra of global dimension $\leq 3$ is quasihereditary.

Example 2.6. The serial algebra A from Dlab \& Ringel (1989, Example, page 283) is of global dimension 4 but is not quasi-stratified.

It is shown in Dlab \& Ringel (1989) that the same algebra is obtained as the endomorphism algebra of a tilting module over a quasihereditary algebra. 
In light of the remark after Corollary 2.3, this fact also shows that the class of quasi-stratified algebras is not closed under tilting (cf. Proposition 6.1, below).

\section{On the Yamagata families of algebras.}

The article Yamagata (1994) gives a method to construct families $\left\{A_{n}\right\}_{n \geq 0}$ of algebras so that the algebras in the family share a set of idempotents, they all have the same Cartan determinant and, if gl. dim. $A_{0}<\infty$, all the algebras have finite, but strictly increasing, global dimension. More information is available about the global dimensions in Yamagata (1994). Our purpose is to show that in any such family $\left\{A_{n}\right\}_{n>0}$, if some $A_{n}$ is quasi-stratified, so is $A_{n+1}$.

In Yamagata's construction, the Loewy length of the algebras strictly increases in passing from $A_{n}$ to $A_{n+1}$. In Kirkman \& Kuzmanovich (1990) there is a construction of a family $\left\{A_{n}\right\}_{n \geq 1}$ of algebras each with two primitive idempotents and each with Loewy length 4 . These are all of finite global dimension but, even at $n=2$, are not quasi-stratified. Cellular algebras (see König \& $\mathrm{Xi}(2005))$ share with quasi-stratified algebras the property that their Cartan determinants are positive and they are ultimate-hereditary exactly when the Cartan determinant is 1 (in fact, the cellular algebras of Cartan determinant one are even quasihereditary). However, the algebra $A$ in the example after König \& Xi (2005, Proposition 4.2) is cellular but not quasi-stratified.

The Yamagata construction begins with an algebra $A_{0}$ and a decomposition of ${ }_{A} A_{0}=P_{0,1} \oplus \cdots \oplus P_{0, m}$. Then, it is assumed that there is a sequence of algebras $A_{0}, A_{1}, A_{2}, \ldots$ with decompositions ${ }_{A} A_{n}=P_{n, 1} \oplus \cdots \oplus P_{n, m}$ and, for $n>0$, algebra surjections $p_{n}: A_{n} \rightarrow A_{n-1}$ so that $\left.p_{n}\right|_{P_{n, i}}=p_{n, i}: P_{n, i} \rightarrow P_{n-1, i}$, $i=1, \ldots, m$. Moreover, the following two conditions are assumed for $n>0$.

(I) There are exact sequences of $A_{n}$-modules

$$
0 \rightarrow P_{n, i+1}^{\alpha_{n, i}} \stackrel{q_{n, i}}{\longrightarrow} P_{n, i} \stackrel{p_{n, i}}{\longrightarrow} P_{n-1, i} \rightarrow 0,
$$

for $i=1, \ldots, m-1$, and

$$
0 \rightarrow P_{n-1,1}^{\alpha_{m, n}} \stackrel{q_{n, m}}{\longrightarrow} P_{n, m} \stackrel{p_{n, m}}{\longrightarrow} P_{n-1, m} \rightarrow 0 .
$$

(II) For $i=1, \ldots, m$, the image of $q_{n, i}$ is in $\mathbf{J} P_{n, i}$ and, by restricting the codomain, $q_{n, i}^{\prime}$ : $\operatorname{ker} p_{n, i} \rightarrow \mathbf{J} P_{n, i}$ gives a split exact sequence

$$
0 \rightarrow \operatorname{ker} p_{n, i} \stackrel{q_{n, i}^{\prime}}{\longrightarrow} \mathbf{J} P_{n, i} \stackrel{r_{n, i}^{\prime}}{\longrightarrow} P_{n-1, i} \rightarrow 0,
$$

with splitting $r_{n, i}^{\prime}: P_{n-1, i} \rightarrow \mathbf{J} P_{n, i}$. The homomorphisms $r_{n, i}^{\prime}$ and $q_{n, m}$ are as $A_{n}$-modules.

It should be noted that the idempotents corresponding to the decompositions of the algebras, denoted $e_{1}, \ldots, e_{m}$ in all the algebras $A_{n}$, are not required to be primitive.

Theorem 3.1. Let $\left\{A_{n}\right\}_{n \geq 0}$ be a family of algebras satisfying the two Yamagata conditions, (I) and (II). If, for any $n>0, A_{n-1}$ is quasi-stratified, so is $A_{n}$. In particular, if $A_{0}$ is quasi-stratified, so are all $A_{n}, n \geq 0$; if $A_{0}$ is quasi-stratified of finite global dimension then each $A_{n}$ is ultimate-hereditary. 
Proof. We will see that $A_{n}$ has nilpotent ideals $I_{1} \subseteq I_{2}$ so that $I_{1}$ is left $A_{n}$-projective, $I_{2} / I_{1}$ is left $A_{n} / I_{1}$-projective and that $I_{2}=\operatorname{ker} p_{n}$. Since the two ideals are nilpotent, they are quasi-stratifying. Then it is only necessary to continue the quasi-stratification using a quasi-stratification of $A_{n} / I_{2}=A_{n-1}$.

We put $I_{1}=\bigoplus_{j=1}^{m-1} \operatorname{ker} p_{n, j}$. By property (I) this is a projective left ideal. It must be shown that it is an ideal. For $a_{j} \in A e_{j}, j<k, p_{n}\left(I_{1} a e_{j}\right)=\mathbf{0}$ and it follows that $I_{1} a e_{j} \subseteq \operatorname{ker} p_{n, j} \subseteq I_{1}$. We also need that $I_{1} A e_{m}=\mathbf{0}$. However, $I_{1} A e_{m} \subseteq \mathbf{J} P_{m . n} \cong P_{n-1, m} \oplus P_{n-1,1}^{\alpha_{m, n}}$, as left $A_{n}$-modules, by (I) and (II). Hence, $I_{1} A e_{m} \subseteq\left(\operatorname{ker} p_{n}\right) \mathbf{J} P_{m, n}=\mathbf{0}$.

We write ${ }^{-}$for "modulo $I_{1}$ ". The candidate for $I_{2}$ is $I_{1}+\operatorname{ker} p_{n, m}=\operatorname{ker} p_{n}$. We have that $\overline{P_{n, i}}=\overline{A_{n}} \overline{e_{i}} \cong P_{n-1, i}$, for $i=1, \ldots, m-1$. Since $I_{1} \cap A_{n} e_{m}=\mathbf{0}$, $\overline{A_{n}}=\overline{A_{n}} \overline{\left(1-e_{m}\right)} \oplus A_{n} e_{m} \cong \bigoplus_{i=1}^{m-1} P_{n-1, i} \oplus P_{n, m}$, as $\overline{A_{n}}$-modules. Hence, ker $p_{n, m} \cong P_{n-1,1}^{\alpha_{m, n}}$ is $\overline{A_{n}}$-projective.

We further need that $\bar{I}_{2}=\overline{\operatorname{ker} p_{n}}$ is an ideal of $\bar{A}_{n}$. However, this follows since $I_{1} \subseteq I_{2}$ and $I_{2}=\operatorname{ker} p_{n}$ is an ideal of $A_{n}$.

If $A_{n}$ is quasi-stratified then the quasi-stratifying chain for $A_{n+1}$ begins with two nilpotent ideals. In fact, if $A_{0}$ is quasi-stratified, then $A_{n}, n>0$, has a quasi-stratifying chain starting with $2 n$ nilpotent ideals.

All the algebras given in Section 3 of Yamagata (1994) are quasi-stratified since they all begin with $A_{0}$ quasi-stratified. In fact they are left ultimatehereditary because their starting points are hereditary.

\section{Elementary algebras - two constructions.}

Throughout this section we have an elementary algebra $A=K \Gamma / I$, where $K$ is a field, $\Gamma$ a finite quiver and $I$ an admissible ideal (see, e.g. Auslander et al. (1997, page 65)). The purpose of this section is to show how to build quasi-stratified algebra from a given one.

In Liu \& Paquette (2006, Example 1.9), the authors use a construction for a specific purpose in which an idempotent is added. The construction is attractive because it illustrates two aspects of the Lui-Paquette work, that some of the ideals in a quasi-stratifying chain may be nilpotent and that projectivity is not only on one side. For that reason, it is worthwhile to generalize it.

Construction 1. We fix an elementary algebra $A=K \Gamma / I$, as above. Two sets of vertices, $\left\{a_{1}, \ldots, a_{s}\right\}$ and $\left\{b_{1}, \ldots, b_{t}\right\}$ are chosen (they do not need to be disjoint). A new vertex $x$ is added to $\Gamma$ along with new arrows $\alpha_{i}: a_{i} \rightarrow x$, $i=1, \ldots, s$ and $\beta_{j}: x \rightarrow b_{j}, j=1, \ldots, t$ to form a quiver $\Gamma^{\prime}$. The new admissible ideal, $I^{\prime}$, is generated as follows: (i) $I \subseteq I^{\prime}$ (ii) for $i=1, \ldots, s$ and $j=1, \ldots, t$, $\beta_{j} \alpha_{i} \in I^{\prime}$, and (iii) for $i=1, \ldots, s$, a set, possibly empty, $L_{i}$ is chosen of elements of the form $r_{i k}=e_{a_{i}} r_{i k} e_{c} \in K \Gamma \backslash I$, each $r_{i k}$ a $K$-linear combination of paths of length $>0$ starting at some vertex $c$; then, all the elements $\alpha_{i} r_{i k}$ are in $I^{\prime}$. We now define $B=K \Gamma^{\prime} / I^{\prime}$. It is clear that $I^{\prime}$ is an admissible ideal and that $B$ is $K$-finite dimensional (any path in $\Gamma^{\prime}$ which goes through $x$ more than twice is in $\left.I^{\prime}\right)$. There are several claims.

Claim 1. $U=\sum_{j=1}^{t} B\left(\beta_{j}+I^{\prime}\right) B=\sum_{j=1}^{t} B\left(\beta_{j}+I^{\prime}\right)$ is projective and $U^{2}=\mathbf{0}$. 
Proof. The only arrows which can precede a $\beta_{j}$ is one of the $\alpha_{i}$; however $\beta_{j} \alpha_{i} \in I^{\prime}$. Hence, we have the first part of the claim. We notice also that for each $j=1, \ldots, t, B\left(\beta_{j}+I^{\prime}\right) \cong B e_{b_{j}}$ because, in the set of generators for $I^{\prime}$ given above, no relation starts in $\beta_{j}$. The same observation shows that the sum $\sum_{j=1}^{t} B\left(\beta_{j}+I^{\prime}\right)$ is direct. Hence, we have the projectivity. Since any path passing first through $\beta_{j}$ and then through $\beta_{k}, 1 \leq j \leq k \leq t$, would contain some $\beta_{k} \alpha_{i}$, some $i$; it would be in $I^{\prime}$. This shows that $U^{2}=\mathbf{0}$.

Claim 2. $V=\sum_{i=1}^{s} B\left(\alpha_{i}+I^{\prime}\right) B=\sum_{i=1}^{s}\left(\alpha_{i}+I^{\prime}\right) B$ and $V_{B}$ is not projective if any of the sets $L_{i}$ is non-empty. This claim is not used to get the conclusion but shows that there need not be left-right symmetry.

Claim 3. We put $\bar{B}=B / U$. Then, $\bar{B} e_{x} \bar{B}=e_{x} \bar{B}$ is projective and $e_{x} \mathbf{J}(\bar{B}) e_{x}=\mathbf{0}$. I.e., $\bar{B} e_{x} \bar{B}$ is a heredity ideal.

Proof. The algebra $\bar{B}$ can be viewed as an elementary algebra $K \Gamma^{\prime \prime} / I^{\prime \prime}$, where $\Gamma^{\prime \prime}$ is obtained from $\Gamma^{\prime}$ by removing the arrows $\beta_{j}, j=1, \ldots, t$, and $I^{\prime \prime}$ is generated by $I$ and the relations in (iii), above. The vertex $x$ is now a sink in $\Gamma^{\prime \prime}$ and $\bar{B} e_{x} \bar{B}=e_{x} \bar{B}$, which is $\bar{B}$ projective. Since the only arrows leaving $x$ are the $\beta_{j}, e_{x} \mathbf{J}(\bar{B}) e_{x}=\mathbf{0}$.

Claim 4. $B /\left(U+B e_{x} B\right)=A$.

Proof. We already have that $\bar{B}=K \Gamma^{\prime \prime} / I^{\prime \prime}$ and that $x$ is a sink in $\Gamma^{\prime \prime}$. Then, as in Corollary 1.6, $\bar{B} / \bar{B} e_{x} \bar{B}=\left(1-e_{x}\right) \bar{B}\left(1-e_{x}\right)=A$.

What has been shown is that, with the data above, if $A$ is quasi-stratified (ultimate-hereditary) then $B$ is quasi-stratified (ultimate-hereditary).

In the above construction, multiple arrows $a_{i} \rightarrow x$ and $x \rightarrow b_{j}$ may be introduced without changing the conclusions.

Construction 2. We use the same notation as above. In this case we produce a new algebra $B$ with an ideal $L$ so that $L^{2}=\mathbf{0},{ }_{B} L$ is projective and $B / L=A$.

We pick two distinct vertices $a$ and $b$ from $\Gamma$ and add a new arrow $\alpha: a \rightarrow b$ to form the quiver $\Gamma^{\prime}$. An ideal of relations is generated in $K \Gamma^{\prime}$ by $I$ and $\{\alpha \lambda \mid t(\lambda)=a\}$. The new algebra is $B=K \Gamma^{\prime} / I^{\prime}$. This ideal $I^{\prime}$ is clearly admissible since any path of sufficient length either has a factor in $I$ or it will contain $\alpha$ twice and be in $I^{\prime}$.

We now consider the ideal $L=B\left(\alpha+I^{\prime}\right) B$. It is easy to verify that (1) $L=$ $B\left(\alpha+I^{\prime}\right),(2) L \cong B e_{b},(3) L^{2}=\mathbf{0}$ and (4) $B / L=A$.

The construction of a square zero ideal projective on the right is similar.

The two constructions show how increasingly complex quasi-stratified and ultimate-hereditary elementary algebras may be built.

\section{Monomial algebras and quadratic monomial algebras.}

In this section we will be looking at finite dimensional monomial algebras, that is, elementary algebras of the form $A=K \Gamma / I$, where $K$ is a field, $\Gamma$ is a finite quiver and $I$ is an admissible ideal generated by paths of length $\geq 2$. For such an algebra we will write $I=\langle\rho\rangle$, where $\rho$ is a minimal generating set which is a set of paths. The minimal projective resolutions of the simple $A$-modules can be constructed algorithmically by looking at certain overlaps of the relations in $\rho$. Details of this method are found in Green et al. (1985) and Anick \& Green (1987). 
The first result is a characterization of when an ideal of the form $A p A, p \notin I$, a path, is left or right projective. (When it is clear from context what is intended, we write $p$ for $p+I$.) It should be noted that a projective nilpotent ideal in a monomial algebra need not be one generated by a path. The proposition is for ${ }_{A} A p A$ projective but the formulation of the right hand version is clear.

Proposition 5.1. Let $A=K \Gamma / I$ be a monomial algebra.

(i) For a vertex $i,{ }_{A} A e_{i} A$ is projective if and only if for any path $q=e_{i} q \notin I$, with length $(q)>0$ and $q$ has no factorization $q_{1} e_{i} q_{2}$ with length $\left(q_{1}\right)>0$, there is no relation $r_{1} r_{2} \in \rho$, where length $\left(r_{2}\right)>0$ and $r_{2}$ is a left factor of $q$.

(ii) For a path $p \notin I$ with length $(p)>0,{ }_{A} A p A$ is projective if and only if for a path $q$ where $p q \notin I$ and $q$ does not have $p$ as a factor, there is no relation $r_{1} r_{2} \in \rho$ where length $\left(r_{2}\right)>0$ and $r_{2}$ is a left factor of $p q$.

Proof. We prove (ii) since the two parts are essentially the same except for some changes in wording. We set $S=\{q$ a path $\mid p q \notin I$ and $q$ has no factor $p\}$. Because $A$ is a monomial algebra, we have $A p A=\bigoplus_{q \in S} A p q$.

$(\Leftarrow)$ : Given a path $q \in S$, then $A p q \cong A e_{t(p)}$; this is because if $A e_{t(p)} \rightarrow A p q$, given by right multiplication by $p q$, had a non-trivial kernel, there would be a relation of the excluded type.

$(\Rightarrow)$ : in this direction we need only observe that each $A p q, q \in S$, is projective. If $q=q e_{i}$, then the condition says that $A p q \cong A e_{i}$ via right multiplication by $p q$.

Monomial algebras where all the relations in $\rho$ are of length 2 are called quadratic monomial algebras. They come up in various contexts. Proposition 5.1 applied to this case gives an interesting result.

Definition 5.2 (Holm (2005)). If $A=K \Gamma / I$ is a quadratic monomial algebra, an oriented cycle $\alpha_{k}, \ldots, \alpha_{1}$, given by its arrows, in $\Gamma$ is said to have full relations if $\alpha_{1} \alpha_{2}, \ldots, \alpha_{k-1} \alpha_{k}$ and $\alpha_{k} \alpha_{1}$ are in $\rho$.

Proposition 5.3. Let $A=K \Gamma / I$ be a quadratic monomial algebra. (i) If gl. $\operatorname{dim} . A<\infty$ then $A$ is left (and right) ultimate-hereditary. (ii) If gl. $\operatorname{dim} . A=$ $\infty$ then there is a chain of ideals $\mathbf{0}=I_{0} \subset I_{1} \subset \cdots \subset I_{k} \subset I_{k+1}=A$, where, for each $j=1, \ldots, k, I_{j} / I_{j-1}$ is nilpotent and left or right $A / I_{j-1}$ projective. Moreover, $B=A / I_{k}$ is quadratic monomial and contains an oriented cycle with full relations.

Proof. (i) We may assume that gl. $\operatorname{dim} . A \geq 3$ since if gl. $\operatorname{dim} . A \leq 2$ then $A$ is quasi-hereditary (Dlab \& Ringel (1989)). Suppose that there are relations in $\rho$ of the form $\alpha_{1} \alpha_{2}, \alpha_{2} \alpha_{3}, \ldots, \alpha_{k-1} \alpha_{k}$ but with no relations of the form $\beta \alpha_{1}$ or $\alpha_{k} \gamma$. By Green et al. (1985), the global dimension is determined by such overlaps and $k$ cannot exceed the global dimension. More exactly, these overlapping relations yield a $k+1$-chain in the sense of Anick \& Green (1987, Definition 2.5) (the conditions of the definition are readily verified for these relations of length 2 ). For the same reason, all the arrows in the set of $k$ relations are distinct. The next step is to show that ${ }_{A} A \alpha_{1} A$ (and, similarly, $A \alpha_{k} A_{A}$ ) is projective. 
If ${ }_{A} A \alpha_{1} A$ is not projective, then the criterion (ii) of Proposition 5.1 fails. This says that there is a relation which, by the nature of $\rho$, must be of the form $\beta \alpha_{1}$, for some arrow $\beta$. However, this would contradict the assumption about the set of relations.

The algebra $A / A \alpha_{1} A$ is also of finite global dimension (Theorem $1.3 \mathrm{I}$ ) and it is a quadratic monomial algebra. The process can continue until we arrive at a factor algebra where the set of generating relations has no overlaps, that is, at a hereditary algebra.

(ii) Even if gl. $\operatorname{dim} . A=\infty$, the process described in the proof of (i) may proceed if there is a set of relations with overlaps, $\alpha_{1} \alpha_{2}, \ldots, \alpha_{k-1} \alpha_{k}$ so that the list cannot be extended with $\beta \alpha_{1} \in \rho$ or $\alpha_{k} \gamma \in \rho$. If some arrow appears more than once in the list, we will already have an oriented cycle with full relations, and, hence, we can assume that the arrows in the list are distinct. Then $I_{1}=A \alpha_{1} A$ or $I_{1}=A \alpha_{k} A$ is a nilpotent ideal projective on one side. By Theorem $1.3 \mathrm{I}$, gl. $\operatorname{dim} . A / I_{1}=\infty$. This process continues until there is no longer a set of overlapping relations which cannot be extended to the left or to the right. We call the resulting quadratic monomial algebra $B$. Since gl. $\operatorname{dim} . B=\infty, Q_{l}(B)$ must contain an oriented cycle and, since the algebra is finite dimensional, such a cycle has a relation, say $\alpha_{1} \alpha_{2}$. This relation has an overlap, say $\alpha_{2} \alpha_{3}$, and this second relation also has an overlap. We get a set of relations with overlaps, $\alpha_{1} \alpha_{2}, \alpha_{2} \alpha_{3}, \ldots$ which must, eventually, have a repeated arrow, $\alpha_{i} \alpha_{i+1}, \ldots, \alpha_{j-1} \alpha_{j}, \alpha_{j} \alpha_{i}$, giving a cycle with full relations.

Holm (2005) gives a formula for the Cartan determinant of a gentle algebra over a field $K$. Such an algebra is Morita equivalent to a quadratic monomial gentle algebra. To recall: An elementary algebra $A=K \Gamma / I$ is a gentle algebra if (i) Each vertex of $\Gamma$ has in-degree at most 2 and out-degree at most 2; (ii) For each arrow $\alpha$ there is at most one arrow $\beta$ with $\alpha \beta \notin I$ and at most one arrow $\gamma$ with $\gamma \alpha \notin I$; (iii) $A$ is quadratic monomial; and (iv) For each arrow $\alpha$ there is at most one arrow $\beta$ with $t(\beta)=s(\alpha)$ and $\alpha \beta \in I$, and at most one arrow $\gamma$ with $s(\gamma)=t(\alpha)$ and $\gamma \alpha \in I$. (An algebra satisfying the first two properties is called special biserial.) Holm (2005, Theorem 1) says, in particular, that if $A$ is an elementary gentle algebra then $\operatorname{cd} A \geq 0$ and, moreover, $\operatorname{cd} A=1$ if and only if $A$ has no oriented cycle with full relations.

In what follows we will need the observation that if $A=K \Gamma / I$ is a gentle algebra and $\alpha$ is an arrow in $\Gamma$ then $A / A \alpha A$ is also a gentle algebra. It is easily seen that the four conditions remain true in the factor algebra.

Corollary 5.4. Let $A=K \Gamma / I$ be a gentle algebra. Then, gl. dim. $A<\infty$ if and only if $\operatorname{cd} A=1$.

Proof. By Proposition 5.3 (i), if gl. dim. $A<\infty$ then $A$ is ultimate-hereditary and $\operatorname{cd} A=1$. (Of course, this also follows because monomial algebras are positively graded.) In the other direction, if $\operatorname{cd} A=1$, we have, by Theorem $1.3 \mathrm{I}$ that gl. $\operatorname{dim} . A<\infty$ if and only if $\operatorname{gl} . \operatorname{dim} . B<\infty$, where $B$ is the quotient of $A$ described in Proposition 5.3 (ii). Moreover, by Theorem 1.3 (II), cd $A=\operatorname{cd} B=1$. If $Q_{l}(B)$ has no oriented cycle, then gl. dim. $B<\infty$, as re- 
quired. Otherwise, we saw in Proposition 5.3 (ii) that the gentle algebra $B$ has a cycle with full relations, giving that $\operatorname{cd} B \neq 1$, by Holm (2005, Theorem 1), excluding that possibility.

There are gentle algebras which are not quasi-stratified. Indeed if $A$ is gentle and its quiver consists of an oriented cycle with full relations and has more than one vertex then $A$ cannot have a quasi-stratifying ideal. Corollary 5.4 does not extend to more general quadratic monomial algebras as the next example shows. There are also quadratic monomial algebras with negative Cartan determinant.

Example 5.5. There is a quadratic monomial, special biserial algebra A such that gl. $\operatorname{dim} . A=\infty$ but $\mathrm{cd} A=1$.

Proof. The following diagram shows the left structure of an algebra with the stated properties.

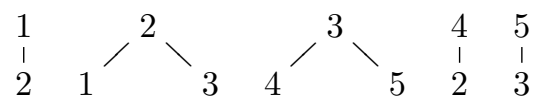

\section{Tilting and cotilting.}

In Colby (1993) it is shown that if ${ }_{A} T$ is a tilting or a cotilting module and $B=\operatorname{End}((T)$, then $\operatorname{cd} A$ and $\operatorname{cd} B$ have the same sign. If, in addition, $A$ is an algebra over an algebraically closed field, then $\operatorname{cd} A=\operatorname{cd} B$. We conclude with the following immediate consequence.

Proposition 6.1. Let $A$ be a quasi-stratified algebra and $B$ an algebra obtained from $A$ by a series of operations of taking endomorphism rings of tilting or cotilting modules. (i) The Cartan determinant conjecture holds for B. (ii) If, in addition, $A$ is an algebra over an algebraically closed field then also the converse of the Cartan determinant conjecture holds for B.

Proof. (i) Since cd $A>0$, then $\mathrm{cd} B>0$ by Colby (1993, Theorem 1).

(ii) If $\operatorname{cd} B=1$ then $\operatorname{cd} A=1$, again by Colby (1993, Theorem 1), and, hence, gl. $\operatorname{dim} . A<\infty$. It follows that all the algebras leading up to $B$ have finite global dimension because finite global dimension is preserved by taking endomorphism rings of tilting or cotilting modules.

\section{Appendix}

In this appendix we sketch the proof of the fact that the class of quasistratified algebras is closed under Morita equivalence. The only surprising aspect is that if we look at an equivalence between categories of left modules, the right projectivity of a quasi-stratifying ideal carries over. The notation and results of Anderson \& Fuller (1992, §21 and $\S 22)$ are used throughout. Let $A$ and $B$ be Morita equivalent algebras (written $A \sim_{M} B$ ) given by an $A-B$ balanced progenerator ${ }_{A} P_{B}$; there are equivalences

$$
\operatorname{Hom}_{A}(P,-): A \text {-Mod } \rightleftarrows B \text {-Mod: } P \otimes_{B}-
$$


which give a lattice isomorphism, $\Phi$ from the lattice of two-sided ideals of $A$ to that of $B$ so that $A / I \sim_{M} B / \Phi(I)$ (Anderson \& Fuller (1992, Proposition 21.11)). More exactly, if $I$ is an ideal of $A$ then $\Phi(I)=\operatorname{Hom}_{A}(P, I P)$. This sends nilpotent ideals to nilpotent ideals (Anderson \& Fuller (1992, Corollary 21.13)) and, by the equivalence $A / I \sim_{M} B / \Phi(I)$, if the idempotent part of $I$ is generated by a primitive idempotent so is that of $\Phi(I)$. (In fact, idempotent ideals are sent to idempotent ideals.) It remains to show that if $I$ is an ideal of $A$ with ${ }_{A} I$ projective then ${ }_{B} \Phi(I)$ is projective and that if $I_{A}$ is projective then $\Phi(I)_{B}$ is projective. The two lemmas are true for rings in general.

Lemma 7.1. Suppose $I$ is an ideal of $A$ with ${ }_{A} I$ projective. Then, ${ }_{A} I P$ is projective and ${ }_{B} \Phi(I)$ is projective.

Proof. First, for any ${ }_{A} M \in A$-Mod,

$$
\operatorname{Hom}_{A}(I P, M) \cong \operatorname{Hom}_{A}\left(I \otimes_{A} P, M\right) \cong \operatorname{Hom}_{A}\left(P, \operatorname{Hom}_{A}(I, M)\right),
$$

by Anderson \& Fuller (1992, Proposition 20.6), showing that $\operatorname{Hom}_{A}(I P,-)$ is exact on $A$-Mod, and it follows that ${ }_{B} \operatorname{Hom}_{A}(P, I P)$ is projective.

The next lemma follows from the left-right symmetry of Morita equivalence but it is interesting to prove it using the given equivalence of $A$-Mod and $B$-Mod.

Lemma 7.2. Suppose $I$ is an ideal of $A$ and $I_{A}$ is projective. Then, $I P_{B}$ is projective and $\Phi(I)_{B}$ is projective.

Proof. If $A^{(X)} \rightarrow I_{A} \rightarrow \mathbf{0}$ splits, so does $A^{(X)} \otimes_{A} P=P^{(X)} \rightarrow I \otimes_{A}$ $P=I P \rightarrow \mathbf{0}$. Moreover (Anderson \& Fuller (1992, Proposition 20.11)), for $M \in \operatorname{Mod}-B$,

$$
\operatorname{Hom}_{B}\left(\operatorname{Hom}_{A}(P, I P), M\right) \cong \operatorname{Hom}_{B}(I P, M) \otimes_{A} P,
$$

so that $\operatorname{Hom}_{B}\left(\operatorname{Hom}_{A}(P, I P),-\right)$ is exact on Mod- $B$.

Theorem 7.3. Let $A$ and $B$ be artin algebras with $A \sim_{M} B$. Then, $A$ is quasi-stratified if and only if $B$ is quasi-stratified.

Proof. Given a stratifying chain for $A$, it suffices to apply one of the two lemmas and the remarks preceding them to each step in the chain.

\section{References}

I. Ágoston, V. Dlab, \& E. Lukács (1998). Stratified algebras, C. R. Math. Acad. Sci. Soc. R. Can. 20, 22-28.

F.W. Anderson and K.R. Fuller (1992). Ring and Categories of Modules, Second Edition, Springer-Verlag, New York, 1992.

D. Anick and E. Green (1987). On the homology of quotients of path algebras, Comm. Algebra 15, 309-341. 
M. Auslander, I. Reiten and S. Smalø (1997). Representations Theory of Artin Algebras, second printing, Cambridge Studies in Advanced Mathematics, 36 Cambridge University Press, Cambridge.

W.D. Burgess and K.R. Fuller (1989). On quasihereditary rings, Proc. Amer. Math. Soc., 106, 321-328.

W.D. Burgess, K.R. Fuller, E.R. Voss and B. Zimmermann-Huisgen (1985). The Cartan matrix as an indicator of finite global dimension for artinian rings, Proc. Amer. Math. Soc. 95, 157-165.

R.R. Colby (1993). Tilting and the Cartan determinant, in Methods in Module Theory, Lecture Notes in Pure and Applied Math. 140, Dekker, New York, $29-31$.

V. Dlab and C.M. Ringel (1989). Quasi-hereditary algebras, Illinois J. Math. 33, 280-291.

K.R. Fuller (1987). Algebras from diagrams, J. Pure Appl. Algebra 48, 23-37.

K.R. Fuller (1992). The Cartan determinant and global dimension of artinian rings, Contemp. Math. 124, 51-72.

E.L. Green, D. Happel and D. Zacharia (1985). Projective resolutions over artin algebras with zero relations, Illinois J. Math. 29, 180-190.

T. Holm (2005). Cartan determinants for gentle algebras, Arch. Math. 85, 233239.

E. Kirkman and J. Kuzmanovich (1990). Algebras with large homological dimensions, Proc. Amer. Math. Soc. 109, 903-906.

S. König and C.C. Xi (2005). When is a cellular algebra quasi-hereditary? typescript.

S.-P. Liu and C. Paquette (2006). Some homological conjectures for quasistratified algebras, J. Algebra 301, 240-255.

K. Yamagata (1994). A construction of algebras with large global dimensions, J. Algebra 163, 51-67. 